\title{
Factors influencing follow-up care post- TIA and minor stroke: a qualitative study using the theoretical domains framework
}

\author{
Grace M. Turner ${ }^{1,2,3^{*}}$, Maria Raisa Jessica V. Aquino ${ }^{4,5}$, Lou Atkins ${ }^{6}$, Robbie Foy ${ }^{7}$, Jonathan Mant ${ }^{5}$ and \\ Melanie Calvert ${ }^{1,2,3,8,9,10}$
}

\begin{abstract}
Background: Follow-up care after transient ischaemic attack (TIA) and minor stroke has been found to be sub-optimal, with individuals often feeling abandoned. We aimed to explore factors influencing holistic follow-up care after TIA and minor stroke.

Methods: Qualitative semi-structured interviews with 24 healthcare providers (HCPs): 5 stroke doctors, 4 nurses, 9 allied health professionals and 6 general practitioners. Participants were recruited from three TIA clinics, seven general practices and one community care trust in the West Midlands, England. Interview transcripts were deductively coded using the Theoretical Domains Framework and themes were generated from coded data.

Results: There was no clear pathway for supporting people with TIA or minor stroke after rapid specialist review in hospital; consequently, these patients had limited access to HCPs from all settings ('Environmental context and resources'). There was lack of understanding of potential needs post-TIA/minor stroke, in particular residual problems such as anxiety/fatigue ('Knowledge'). Identification and management of needs was largely influenced by HCPs' perceived role, professional training ('Social professional role and identity') and time constraints ('Environmental context and resources'). Follow-up was often passive - with onerous on patients to seek support - and predominantly focused on acute medical management ('Intentions'/'Goal').
\end{abstract}

Conclusions: Follow-up care post-TIA/minor stroke is currently sub-optimal. Through identifying factors which influence follow-up, we can inform guidelines and practical strategies to improve holistic healthcare.

Keywords: Transient ischaemic attack, TIA, Minor stroke, Follow-up, Theoretical domains framework

\section{Introduction}

Transient ischaemic attack (TIA) and minor stroke are important risk factors for major stroke. Over 46,000 people experience a first TIA or minor stroke per year and half a million people live with TIA and/or minor stroke in the United Kingdom [1].

\footnotetext{
*Correspondence: G.Turner.1@bham.ac.uk

${ }^{3}$ NIHR Surgical Reconstruction and Microbiology Research Centre, University Hospitals Birmingham NHS Foundation Trust and University of Birmingham, B15 2TH, Birmingham, UK

Full list of author information is available at the end of the article
}

National guidelines promote rapid diagnosis and longterm management that focuses on stroke prevention [2-4]. However, many people with TIA and minor stroke feel unsupported in stroke prevention - both medication and lifestyle change - and often lack basic understanding of their diagnosis, stroke risk and preventative medication [5]. Furthermore, many people experience important residual impairments post-TIA and minor stroke; including, fatigue, psychological and cognitive problems [5-9]. These impairments can affect quality of life, ability to return to work or usual activities, and relationships with 
family and friends [5, 10-15]. Therefore, person-centred care after TIA and minor stroke should be multifaceted and encompass (i) information provision (diagnosis and stroke risk); (ii) stroke prevention (medication and lifestyle change); and (iii) holistic needs (residual problems and return to work and usual activities) $[5,16]$. However, follow-up care is reported to be inadequate and variable with patients feeling abandoned after specialist review [5].

Several studies have explored TIA and minor stroke patients' perspectives of initial treatment and follow-up $[10,14,16]$; however, none have investigated healthcare providers (HCPs) experiences. To improve future care, it is important to identify gaps in follow-up healthcare provision and understand influences on related HCP behaviours. This qualitative study aimed to explore factors influencing holistic follow-up care post-TIA/ minor stroke among different HCPs (doctors, nurses, allied health professionals [AHPs]) and across different health care settings (primary, secondary and community care).

\section{Methods}

Study design

Qualitative, theory-guided, semi-structured interviews. A qualitative approach was selected as this study focuses on exploring attitudes and perceptions of HCPs. Semistructured interviews were preferred to focus groups because it allowed the researcher to obtain richer and deeper data.

\section{Theoretical domains framework}

Changing clinical practice requires an understanding of the influences on behaviour; therefore, our study was underpinned by the Theoretical Domains Framework (TDF) [17]. This theoretical framework was developed for implementation research to identify influences on HCP behaviour [17]. We used the 14-domain TDF (v2) (see Additional file 1: Appendix eTable 1). The first stage of TDF-based research is to select and specify the target behaviour(s) [17]. In our study, target behaviours were (i) identification and (ii) management of individuals' needs post-TIA/ minor stroke. 'Needs' were informed by the literature and defined as: information provision (diagnosis and stroke risk); supporting stroke prevention (medication and lifestyle change); and addressing holistic needs (residual problems and return to work or usual activities).

\section{Participants}

Participants were HCPs who work with TIA or minor stroke patients, including: secondary care doctors, nurses or AHPs; primary care general practitioners [GPs]; and community care AHPs and nurses. Convenience and snowball sampling were used initially; however, sampling became increasingly purposeful to achieve variation in clinical role (doctor, nurse, AHP) and setting (primary, secondary, community care). Secondary care doctors, nurses and AHPs were recruited from three TIA clinics in the West Midlands (England). Community HCPs were recruited from Birmingham Community Healthcare Trust. GPs were recruited from two general practices in the West Midlands and through snowballing. A pragmatic approach to site selection was used whereby research invitations were sent to potential sites and those that responded first were selected. Recruitment rates (percentage of HCPs interviewed of those invited to participate) were: $86 \%(12 / 16)$ for secondary care, $60 \%(6 / 10)$ for community care and $43 \%$ (6/14) for GPs.

\section{Data collection}

One-to-one, semi-structured interviews were conducted by telephone or face-to-face (at the University of Birmingham or participants' workplace). All interviews were conducted by GT, a female, non-medical researcher trained in qualitative research methods. All participants were interviewed once. The interviewer did not have a relationship with any of the participants.

Topic guides were informed by existing literature and consultation with the research team and patient partners, and refined through piloting with patient partners. Topic guides covered: follow-up pathways and communication between healthcare settings, and current practice to identify and address needs. Participants also completed a short demographic questionnaire. Fieldnotes were taken during and after each interview to add contextual information, and reflect on how the interview was conducted. Adjustments were made if necessary during the following interviews, thereby improving reflexivity and trustworthiness of the data.

Digital audio recorded interviews were transcribed verbatim by a professional transcription service. Interviews were conducted between March and November 2018, until the research team judged that the sample and data had sufficient depth and breadth to address the research questions.

\section{Analysis}

Computer Aided Qualitative Data Analysis Software (CAQDAS) QSR NVivo 12 supported the sorting, coding and organisation of transcribed data. A key advantage of using CAQDAS software is that it facilitates and enhances researcher reflexivity [18]. Transcripts were read several times to enable familiarisation with the interviews. Participant responses were deductively coded into relevant TDF domains. GT coded all transcripts and RA independently coded a subset $(10 \%, n=2)$. GT and RA are both experienced qualitative researchers. 
Responses coded in different domains were discussed by GT and RA to establish a consensus; all differences in coding were resolved. Themes were iteratively generated from coded data. Initial data coding and generation of themes was concurrent with the interviews and data collection stopped when no new themes were identified. Themes were identified initially by GT, reviewed by RA, and developed and refined by discussion between GT and RA. The wider research team and our patient partners met on a regular basis to discuss and further refine the themes.

\section{Ethical approval and participants' informed consent}

Ethical approval was given by the Warwickshire North West - Greater Manchester East Research Ethics Committee (Reference: 17/NW/0737). Written or recorded verbal informed consent was obtained from the participants, by the interviewer, immediately prior to the interview. All methods were carried out in accordance with the UK Policy Framework for Health and Social Care Research and the Declaration of Helsinki.

\section{Results}

The final sample consisted of 24 HCPs (5 stroke doctors, 4 nurses, 9 AHPs and 6 GPs). Participants' years of experience ranged from 3 to 37 (mean 15, standard deviation 9), characteristics are summarised in Table 1 and detailed in eTable 2 (see Additional file 1: Appendix). Mean interview length was $48 \mathrm{~min}$ (range 34 to $68 \mathrm{~min}$ ).

Eleven TDF domains were identified as relevant: Environmental context and resources; Social influences; Knowledge; Intentions; Memory, attention and decision processes; Social/professional role and identity; Beliefs about capabilities; Beliefs about Consequences; Reinforcement; Skills; and Goals (Tables 2 and 4).

There were 3 overarching themes and 10 sub-themes:

1. Pathways and access to follow-up care (Lack of standardised follow-up care pathway; Interface between healthcare settings; quotes are provided in Table 2).

2. Identifying needs (Approach to identifying needs; Use of checklists and screening tools; Patient factors; quotes are provided in Table 3)

3. Addressing needs (Stroke prevention; Residual impairments; Education about diagnosis, stroke risk and medication; Use of support services and resources; Patient factors; quotes are provided in Table 4)

Themes and subthemes are described below and summarised in Tables 2 and 4 and Fig. 1.
Table 1 Characteristics of participants $(n=24)$

\begin{tabular}{lll}
\hline Variable & & Number (\%) \\
\hline Age (years) & $21-30$ & $1(4.2)$ \\
& $31-40$ & $8(33.3)$ \\
& $41-50$ & $12(50.0)$ \\
Sex & $51-60$ & $3(12.5)$ \\
Profession & Male & $10(41.7)$ \\
& Female & $14(58.3)$ \\
& Stroke consultant ${ }^{\text {a }}$ & $5(20.8)$ \\
Healthcare setting & Nurse & $4(16.7)$ \\
& Allied health professional & $9(37.5)$ \\
& General practitioner & $6(25.0)$ \\
& Secondary care & $9(37.5)$ \\
Years of experience & Community care & $6(25.0)$ \\
& Secondary \& community care & $3(25.0)$ \\
& $5-10$ & $3(12.5)$ \\
& $11-20$ & $5(20.8)$ \\
& $>20$ & $11(45.8)$ \\
\end{tabular}

${ }^{a}$ physicians with specialist skills in stroke medicine

\section{Pathways and access to follow-up care}

\section{Lack of standardised follow-up care pathway}

Specialist review in secondary care is rapid with patients leaving hospital within hours or days. Subsequent follow-up is limited with no standardised follow-up care pathway and variability in follow-up between and within healthcare settings (Environmental context and resources). For example, one hospital had a nurse-led follow-up clinic, but consultants within that hospital differed in whether they referred their patients there or not (Intentions); whereas, another hospital had no follow-up (Environmental context and resources). The eligibility criteria to access early supported discharge varied across regions; minor stroke patients "rarely" received early supported discharge and TIA patients were not eligible (Environmental context and resources). In primary care, there was a spectrum of active vs passive approaches to follow-up with some general practices actively contacting patients and others relying on patients to contact them (Intentions). GPs often relied on annual long-term condition reviews as follow-up for these patients (Environmental context and resources).

\section{Interface between healthcare settings}

Communication between healthcare settings was restricted. It was usually one way and comprised electronic or paper discharge letters which varied in the speed they were sent, the content, and how the 
Table 2 Theoretical domains and sample quotes related to pathways and access to follow-up care

TDF domain $\quad$ Theme (Barrier [B], Enabler [E], Mixed [M]) Quote

\section{Lack of standardised follow-up care pathway}

Environmental context and resources

Variability in follow-up pathways (M)

Restricted access to early supported discharge (B)

Intentions

\section{Interface between healthcare settings}

Environmental context and resources

Intentions

Social Influences
Variability in consultants use of nurse-led followup (M)

Variability in GPs having an active vs passive approach to follow-up (M)

Restricted communication between healthcare

Variability in content and speed of discharge letters (M)

Variability in how GPs engage with discharge letters (M)

GPs have difficulty accessing imaging results and specialist stroke advice (B) settings (B)
"So we found that with some TIA clinics they offer a follow up appointment around six to eight weeks, sometimes it's consultant led and sometimes it's nurse led so you can imagine that those appointments would be very different depending on who they speak to whereas other TIA clinics don't have that option at all so there's a very disjointed follow up pathway which people are getting..." [H8, GP, 17 Years of experience]

"So, for the TIAs obviously we don't do that [Early Supported Discharge] there's no follow up." [H17, Stroke consultant, 8Years of experience]

"We do have a, for follow-up we do have a nurse lead follow-up clinic. Which I have access to, but I don't use a lot. And again, there's some variation in practice amongst the five stroke physicians about how much they use that clinic."

[H2O, Neurologist, 22 Years of experience]

"...personally I quite like to see patients particularly when patients have been started on a whole bunch of new tablets... So, I like to get them to come and see me."

[H11, GP, 18Years of experience]

"... it wouldn't be feasible for every specialist letter we get for strokes and everything else to contact the patient to sort of go through the [medication], we wouldn't do anything else really. So we add the medication to the repeat prescription"

[H13, GP, 13 Years of experience]

"So, I think for me part of the problem is sometimes the access to the specialist. And yeah, we can fax over letters and we can make phone calls and we can try and bleep people and we can email and all this sort of thing, but you know my experience generally is that we don't get a lot of information back." [H11, GP, 18Years of experience]

"Communication is quite good. It's quick. The turnaround on letters is quick..."

[H3, AHP, 23 Years of experience]

"We're still relying on old paper letters, which, you know, we probably shouldn't be anymore and communication is very slow, so it takes us six weeks often to get a clinic letter and if something's urgent then we can't afford to wait that long." [H13, GP, 13 Years of experience]

"And we tend to just wait for the [discharge] letter, act on it... It's very much been directed by the secondary care rather than doing a massive amount off our own backs."

[H12, GP, 7 Years of experience]

"And certainly the discharge letters are quite 'protocolised' again in that... And there's things that they will put on there that you just think 'well, there's no need for that to be on there' in terms of giving me advice here..."

[H11, GP, 18Years of experience]

"But obviously it's a bit hard to give them [patients] absolute reassurance because in terms of the scan reports or the results on the investigations, may not be entirely with us..."

$[\mathrm{H} 9, \mathrm{GP}, 6$ Years of experience] 
Table 3 Theoretical domains and sample quotes related to identifying needs

TDF domain $\quad$ Theme (Barrier [B], Enabler [E], Mixed [M])

\section{Approaches to identifying needs}

Social professional role and identity

Goal

Intentions

Beliefs about capabilities
Perceived role in follow-up care influences approaches to identifying needs (M)

Professional training influenced approaches to identifying needs (M)

Knowledge/ lack of knowledge of potential patient needs (M)

HCPs had different perceptions on the goal of their follow-up (M)

Active vs passive approach to identifying needs (M)

Personal experience of TIA/minor stroke (E)

Confident/ not confident in identifying needs (M)

\section{Quote}

"I: So, do you see that a part of your role to ask about things like people's social activities and their mood and cognition and the more holistic side?

IV: Yeah, I think it is part of our role..."

[H22, nurse, 13 Years of experience]

"Usually it's pretty much a one-stop clinic so if they need a carotid scan they get it there. If they need a brain scan urgently they get it there. We give them the medication that they need to prevent further events, book any other tests which are non-urgent but still need to be done and then we discharge them. So it's a one-stop medical clinic".

[H24, consultant, 12 Years of experience]

"As an OT, obviously we're dual trained in physical and mental health."

[H14, AHP, 17Years of experience]

"...but there might be a lack of education, medical education about the, yeah, the long-term consequences really."

[H13, GP, 13 Years of experience]

"... and that can affect you, you know you can't drive, you maybe can't work, can't watch TV, can't read, it's a very small minor stroke but it's had a big effect." [H4, nurse, 37 Years of experience]

"In the review clinic, we make sure two things, one, that all investigations have been completed. Secondly all the risk factors have been addressed and thirdly they're on the right medications for the conditions. So, we just see them one more time after being seen in the TIA clinic."

[H7, consultant, 20Years of experience]

"And it's quite a holistic type clinic so we look at them although obviously we're focussing on the stroke, we're looking at the whole person."

[H4, nurse, 37Years of experience]

"... so the follow up that I offer tends to be just checking that they're ok, that they're sort of getting on with their medications that they have recently been prescribed and just ensuring that they are kind of informed about you know what the process is and any further results that are coming back through and I guess sort of just general support about you know ongoing risk factors and risk reduction..."

[H8, GP, 17 Years of experience]

"I don't actively ask for it, I don't actively for sleep and emotional problems, not things that I tend to ask about..."

[H20, consultant, 22 Years of experience]

"So, there's an element of tailoring. But we do always generally check mood, fatigue, confidence as well, as part of what we're doing."

[H22, nurse, 13 Years of experience]

".. but it almost seems like there is a kind of post TIA syndrome and certainly I probably first became aware of that through personal experience really rather than in the practice."

[H8, GP, 17 Years of experience]

"I: Do you feel quite confident in being able to identify what their [patients'] needs are? IV: Yeah. yeah, I think I definitely..." [H16, AHP, 4 Years of experience] 
Table 3 (continued)

\begin{tabular}{ll}
\hline TDF domain & Theme (Barrier [B], Enabler [E], Mixed [M]) \\
\hline $\begin{array}{l}\text { Use of checklists and screening tools } \\
\text { Environmental context and resources }\end{array}$ & $\begin{array}{l}\text { Checklists/ screening tools used/ not used to } \\
\text { facilitate identification of needs (M) }\end{array}$
\end{tabular}

Lack of time to use screening tools (B)

\section{Beliefs about consequences}

Reinforcement

Memory, attention and decision processes
Checklist/ screening tools considered useful/ not useful (M)

Content of primary care long-term conditions template is influenced by performance-based incentives (Quality and Outcomes Framework) (B)
Screening tool mandated by local Clinical Commissioning Group (B)

Checklist/ screening tool used to inform decision

\section{Quote}

"We use formal mood screens in [location]... the HADS, the DISC, those types of things. In terms of fatigue we use self-rating scales for fatigue. Obviously, cognition we've got a whole host of standardised assessments that we use, alongside functional assessments as well. Anxiety again, would be self-rating. And fear of falling would be self-rated. We don't use every single one with every single patient but we have those."

[H14, AHP, 17Years of experience] We don't, at the moment, do a formal mood assessment and we don't do a formal cognitive screen within clinic."

[H3, AHP, 23 Years of experience]

"... in a clinic setting, there isn't really time to do lots of formal screening."

[H3, AHP, 23 Years of experience]

"I mean the screen tools don't always pick up on these things and sometimes we've found that, you know, at home they answer that everything's alright on the PHQ's but actually when you see them they are clearly upset about something."

[H8, GP, 17 Years of experience]

"It [checklist] just gets a good, overall idea of what they're doing and then identifies then at the end of it what they need to be referred to."

[H1, AHP, 5 Years of experience]

"Cause one of the things we have at present in our clinical systems is templates... I think they tend to

B) be very much QOF kind of based. So, it'll probably be addressing things like cholesterol, blood pressure, their sugar etc. etc. Medication, making sure they are on the appropriate medications... I don't think it actually addresses the kind of psychological aspects." [H10, GP, 31 Years of experience] "...the Barthel Index is obviously the Clinician Commissioning Group level, so I don't think that will change..."

$[\mathrm{H} 2, \mathrm{AHP}, 3$ Years of experience $]$ making (E)

"... then we'll use it [screening tool] to set the goals and then we're doing it to give them to focus. What we want to do is improve their score and also so that we can monitor that what we're doing is effective as well." [H5, AHP, 16 Years of experience] provide weekend service on the wards. Unfortunately not in ESD at the moment, so, if a physio is working at the weekend, they can do a MOCHA over the weekend, so, there isn't that delay." [H14, AHP, 17 Years of experience] 
Table 3 (continued)

\begin{tabular}{|c|c|c|}
\hline TDF domain & Theme (Barrier [B], Enabler [E], Mixed [M]) & Quote \\
\hline \multicolumn{3}{|l|}{ Patient factors } \\
\hline \multirow[t]{3}{*}{ Social influences } & Cultural/ language barriers (B) & $\begin{array}{l}\text { "The obvious one is language and non-English speak- } \\
\text { ing patients where you may not know that until they } \\
\text { come to clinic and you're really then stuck..." } \\
\text { [H3, AHP, } 23 \text { Years of experience] }\end{array}$ \\
\hline & $\begin{array}{l}\text { Patients not wanting to "bother" doctor or raise } \\
\text { non-medical issues (B) }\end{array}$ & $\begin{array}{l}\text { "... especially elderly people they don't pester their GP } \\
\text { for things, my mum says that, I don't want to trouble } \\
\text { the GP." } \\
{[\mathrm{H} 4 \text {, nurse, } 37 \text { Years of experience }]}\end{array}$ \\
\hline & $\begin{array}{l}\text { Family members as facilitators/ barriers to identifi- } \\
\text { cation of patient needs (M) }\end{array}$ & $\begin{array}{l}\text { "The other one is just normally again partners coming } \\
\text { in and it tends to be men who come in and they don't } \\
\text { say a great deal and then the partner or wife mentions } \\
\text { they're worried that the patient's been like this for a } \\
\text { long time and then they tell me everything." } \\
{[\mathrm{H} 12, \mathrm{GP}, 7 \text { Years of experience] }} \\
\text { "...people can be quite proud and not want to sort of, } \\
\text { they want to put a good front on it for other family } \\
\text { members and things and not admit it." } \\
{[\mathrm{H} 6, \mathrm{HPP}]}\end{array}$ \\
\hline
\end{tabular}

recipient HCP engaged with the letter (Environmental context and resources/ Intentions). Some GPs described frustration with the lack of access to results of imaging/ investigations and difficulty in contacting stroke consultants for specialist advice (Environmental context and resources/ Social influences).

\section{Identifying needs}

\section{Approaches to identifying needs}

HCPs' perceived role in follow-up care (Social professional role and identity) and knowledge of potential patients' needs (Knowledge) influenced their approach to identifying needs. Consultants mostly considered the purpose of their follow-up clinics to be related to the acute management of TIA/ minor stroke, in particular interpreting and actioning imaging and investigations (Goal). For GPs, follow-up was predominantly focused on stroke prevention medication and risk factor management, such as blood pressure control (Goal). GPs and consultants were usually passive and relied on patients to raise issues themselves (Intentions/ Social influences). In contrast, nurses and AHPs took an active approach to identifying needs (Social professional role and identity/ Intentions). In general, nurses and AHPs had a more holistic understanding of potential needs and were confident in their ability to identify needs (Knowledge/ Beliefs about capabilities).

Overall, there was a lack of knowledge and understanding of sequelae post-TIA/ minor stroke, particularly amongst consultants and GPs who often viewed TIA/ minor stroke as transient events (Knowledge). Knowledge of residual impairments post-TIA/ minor stroke was gained through experience of following-up these patients
(Knowledge) or, less commonly, through personal experience with family members (Social influences).

\section{Use of checklists and screening tools}

Checklists, usually developed in-house, were commonly used by nurses and AHPs and considered useful prompts (Environmental context and resources/ Beliefs about consequences). However, checklists were less commonly used by experienced nurses and AHPs who referred to using "instinct" to identify needs, gained through professional experience working in the field (Skill). In primary care, a long-term conditions template is used by nurses at annual reviews. However, the content was medically focused and influenced by a performance-based incentive: the Quality and Outcomes Framework (QOF) (Reinforcement).

AHPs varied considerably in their use of screening tools, if at all (Environmental context and resources). When used, screening tools informed decision making, such as psychology referral (Memory, attention and decision processes). In one early supported discharge team, the Barthel index was mandated by the local service commissioners (Reinforcement); however, this tool was not considered useful by AHPs (Beliefs about consequences). Screening tools were rarely used by GPs and consultants. Barriers to use of screening tools included lack of knowledge of available tools (Knowledge), lack of training to used and interpret tools (Skills) and lack of time (Environmental context and resources).

\section{Patient factors}

Culture and language were sometimes barriers to identifying needs (Social influences). There was a perception 


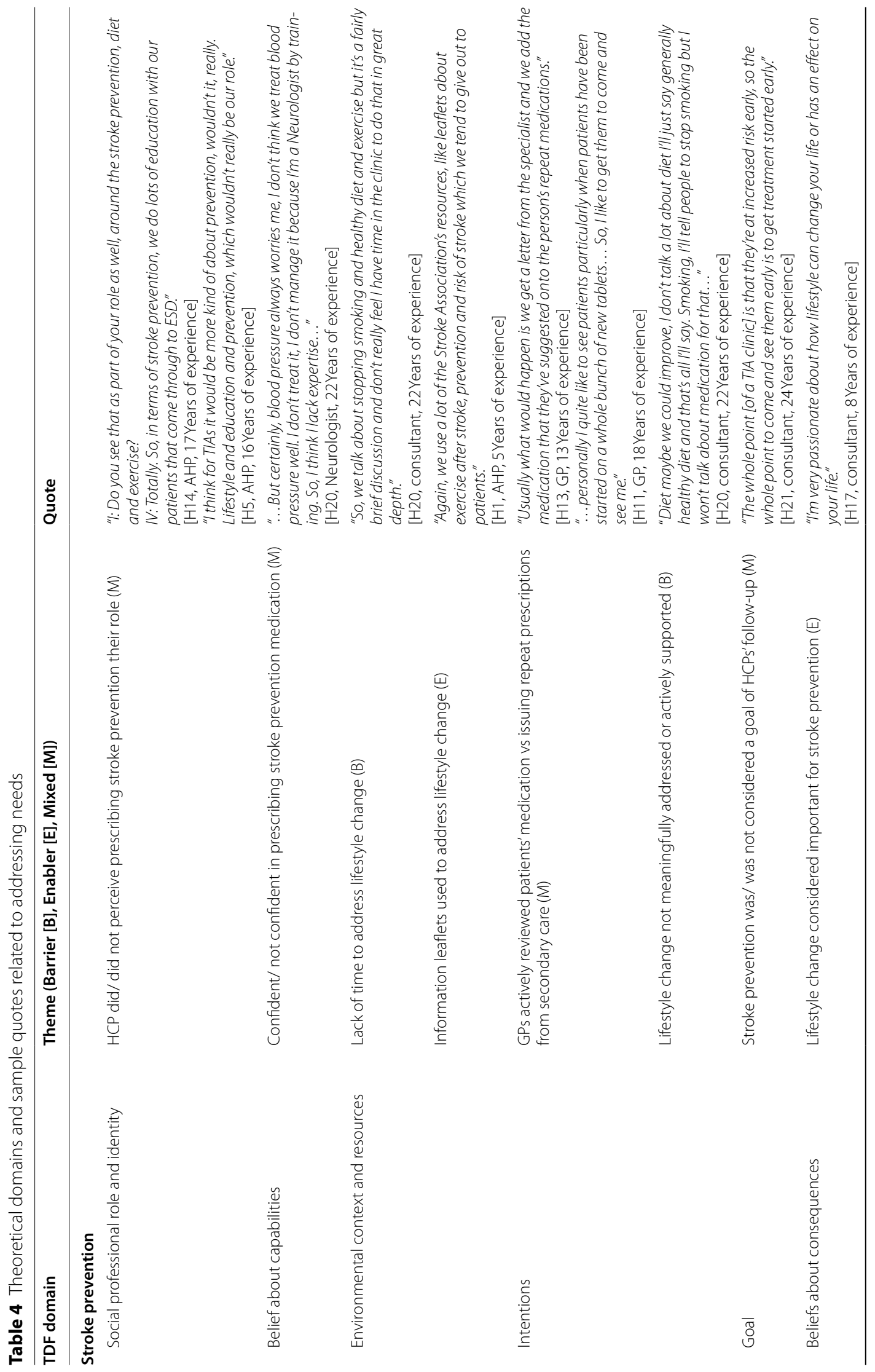




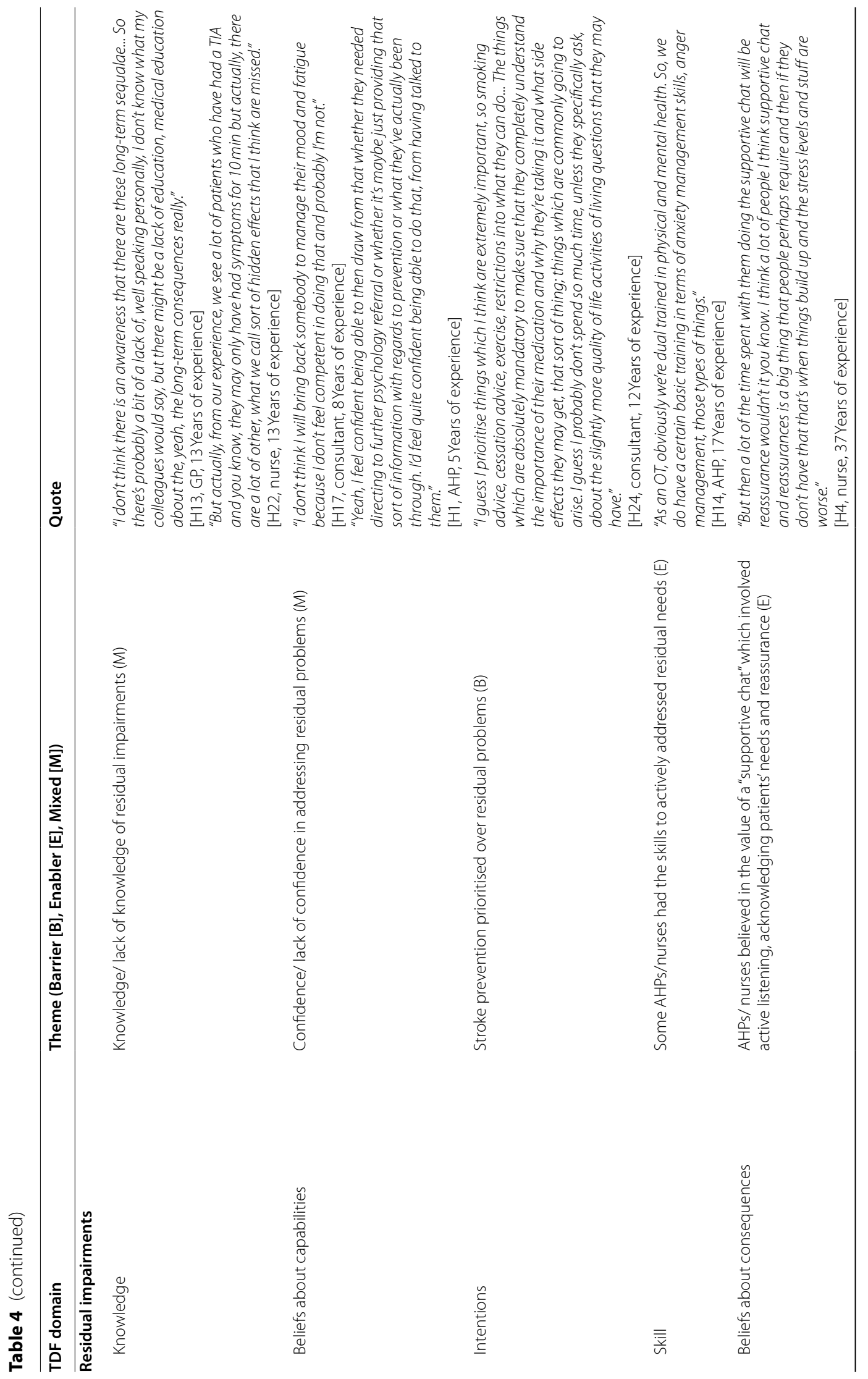




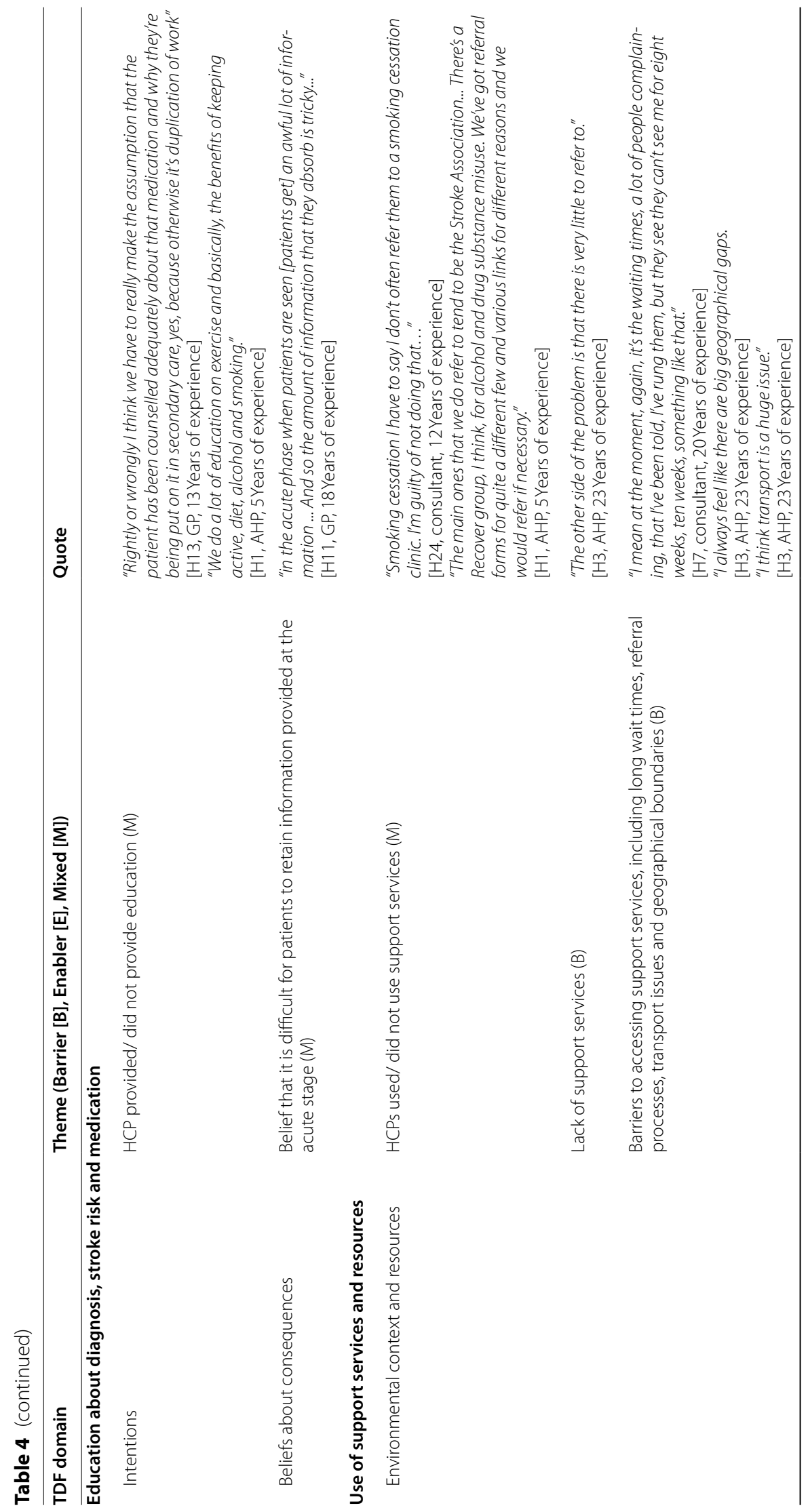




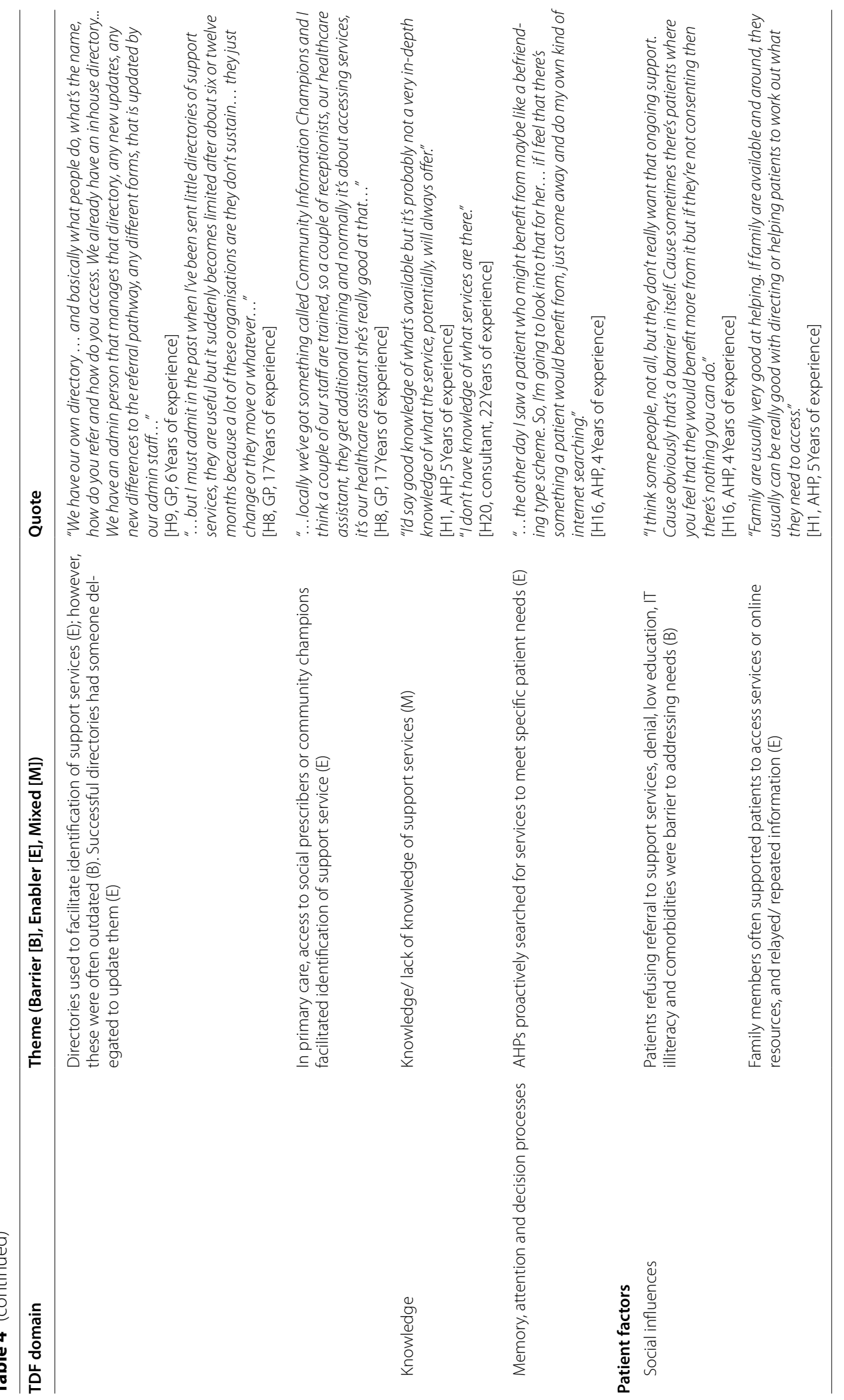




\begin{tabular}{|c|c|c|}
\hline Pathways and access to follow-up care & Identifying needs & Addressing needs \\
\hline $\begin{array}{l}\text { A) Lack of standardised follow-up } \\
\text { pathway } \\
\text { - Hospital: variable follow-up e.g. } \\
\text { consultant- led, nurse-led, none } \\
\text { (ECR) } \\
\text { - Primary care: variability in active } \\
\text { or passive approach to inviting } \\
\text { patients for follow-up (I) } \\
\text { - Community: very limited access } \\
\text { (ECR) } \\
\text { B) Interface between healthcare } \\
\text { settings } \\
\text { - Communication restricted (ECR) } \\
\text { - Discharge letters: Variation in } \\
\text { content/ speed and how GPS used } \\
\text { these letters (ECR/I) } \\
\text { - Primary care: Lack of access to } \\
\text { imaging/ investigations and } \\
\text { specialist stroke advice (ECR/SI) }\end{array}$ & $\begin{array}{l}\text { A) Approaches to identifying needs } \\
\text { - Influenced by HCPs' perceived } \\
\text { - } \text { role in follow-up care (SPRI/G) } \\
\text { - Lackive vs active approach ( } I \text { ) } \\
\text { needs (K) } \\
\text { B) Checklist and screening tools } \\
\text { - Checklist: useful prompts (ECR) } \\
\text { - Screening tools: rarely used by } \\
\text { doctors, occasionally used by } \\
\text { AHPs (ECR) } \\
\text { - Barriers: lack of: knowledge (K), } \\
\text { training (S) and time (ECR) } \\
\text { C) Patient factors (SI) } \\
\text { - Barriers: culture, language, } \\
\text { embarrassment, fear of } \\
\text { consequences e.g. driving } \\
\text { restrictions } \\
\text { - Facilitators: family members }\end{array}$ & $\begin{array}{l}\text { A) Stroke prevention } \\
\text { - Differences in perceived } \\
\text { responsibility for prescribing } \\
\text { medication (SPRI/I) } \\
\text { - Limited time for lifestyle change } \\
\text { (ECR) } \\
\text { B) Residual impairments } \\
\text { - Lack of: knowledge (K) and } \\
\text { confidence in abilities (BCap) } \\
\text { C) Education } \\
\text { - Limited time (ECR) } \\
\text { D) Support services/ resources } \\
\text { - Barriers: lack of services, wait } \\
\text { times, referral processes, transport } \\
\text { (ECR), knowledge (K) } \\
\text { - Facilitators: directories (ECR) } \\
\text { E) Patient factors (SI) } \\
\text { - Barriers: refusing referrals, denial, } \\
\text { IT illiteracy, comorbidities } \\
\text { - Facilitators: support from family }\end{array}$ \\
\hline
\end{tabular}

BCap: Beliefs about capabilities; ECR: Environmental context and resources; G: Goals; I: Intentions; K: Knowledge; S: Skills; SI: Social influences

Fig. 1 Summary of themes and subthemes

that patients do not want to raise non-medical or minor issues with doctors, but are more willing to discuss these problems with nurses and AHPs (Social influences). It was perceived that some patients withheld information due to embarrassment, not wanting to admit to problems for fear of consequences, such as driving restrictions (Social influences).

Family members facilitated identification of needs, in particular, women raised issues on behalf of their male partners (Social influences). On occasion, family members acted as translators (Social influences). However, in some instances, presence of family could be a barrier if patients were embarrassed to discuss problems in front of them (Social influences).

\section{Addressing needs Stroke prevention}

There was disparity between consultants and GPs regarding perceived responsibility for prescribing stroke prevention medication (Social professional role and identity). Some consultants considered this to be part of their role (Social professional role and identity), but varied in tailored versus protocolised approaches to prescribing (Intentions). In contrast, other consultants were less confident prescribers and relied on GPs (Beliefs about capabilities/ Intentions). Some GPs perceived preventative medicine as a strength of general practice and believed prescribing medication was their role, particularly in the context of comorbidities and polypharmacy (Social professional role and identity/ Goal). However, other GPs felt they lacked specialist stroke knowledge and relied on stroke consultants (Belief about capabilities/ Social influences). This influenced whether GPs actively saw patients and reviewed their medication or simply issued repeat prescriptions based on secondary care recommendations (Intentions).

Although HCPs recognised the importance of lifestyle change for stroke prevention (Beliefs about consequences), this was rarely addressed in a meaningful way or actively supported (Intentions). Time constraints were a key barrier (Environmental context and resources); however, behaviours were also influenced by lifestyle change not being considered a key goal of their follow-up (Goal/ Social professional role and identity) and lack of knowledge of support services and resources to support lifestyle change (Knowledge). As a substitute, HCPs used information leaflets and consultants made recommendations to GPs through discharge letters (Environmental context and resources).

\section{Residual impairments}

Consultants rarely addressed residual problems, such as fatigue, psychological and cognitive problems, due 
to lack of knowledge of potential problems and lack of confidence in how to address these problems (Knowledge/ Beliefs about capabilities). Although most GPs lacked understanding of residual problems related to TIA and minor stroke, they were aware of holistic consequences from long-term conditions in general (Knowledge); however, restricted time (typically within 10-min appointments) and prioritisation of stroke prevention partly influenced by performance based financial incentives (QOF) - were commonly cited as reasons for not addressing these issues (Environmental context and resources/ Intentions/ Reinforcement).

AHPs and nurses considered addressing holistic needs part their role and were confident in their ability to address residual needs (Social professional role and identity/ Beliefs about capabilities). Often AHPs and nurses had the skills to actively addressed residual needs, such fatigue management, and tailored their approach to patients' demographic traits and personal circumstances (Skill/ Memory attention and decision processes). Many AHPs and nurses also believed in the value of a "supportive chat" which involved active listening, acknowledging patients' needs and reassurance (Beliefs about consequences) and recognised the importance of lay language (Skill).

\section{Education about diagnosis, stroke risk and medication}

Consultants usually provided information on diagnosis and stroke risk (Intentions); however, this was not comprehensive and there was belief that it is difficult for patients to retain information provided at the acute stage (Beliefs about consequences). Most GPs did not routinely provide education due to time constraints and an assumption that this had been done in secondary care (Environmental context and resources/ Social professional role and identity). Most nurses and AHPs actively provided education and considered this part of their role (Intentions/ Social professional role and identity). They often used stroke charity websites to supplement verbal information (Environmental context and resources).

\section{Use of support services and resources}

Support services were mentioned as potential sources of support, both formal healthcare services, such as smoking cessation and talking therapy, and local community groups (Environmental context and resources). However, barriers to accessing services were reported, including a lack of services, long wait times to access services, lengthy or complicated referral processes, transport issues, and geographical boundaries (Environmental context and resources).
Another important barrier was lack of knowledge of services, particularly as they changed rapidly (Knowledge). In-house directories were sometimes used in primary and community care; however, directories were often outdated unless someone was delegated to regularly update them (Environmental context and resources). In early supported discharge teams, knowledge of support services was facilitated by multidisciplinary team working, building relationships in the community and experience of working in the area (Knowledge). AHPs also proactively searched for services to meet specific patient needs (Memory, attention and decision processes). In primary care, GPs having access to social prescribers or community champions facilitated identification of support services (Environmental context and resources).

\section{Patient factors}

Patient factors were sometimes barriers to addressing needs, including patients refusing referral to support services, denial, low education, IT illiteracy and comorbidities (Social influences). Family members were often facilitators and supported patients to find or access services, access the internet or computers, relaying or repeating information (Social influences).

\section{Discussion}

There is no clear pathway for supporting people with TIA or minor stroke after rapid specialist review in hospital. Consequently, these patients have limited access to HCPs from all settings. HCPs' approaches to identifying and addressing needs are predominantly influenced by their professional training, perceived role and purpose of their clinic, knowledge of potential needs, and time constraints. In general, consultants and GPs are medically focused; whereas, nurses and AHPs are more holistic in their approach to follow-up care. However, TIA/ minor stroke patients are unlikely to receive follow-up from nurses and AHPs.

\section{Comparison with other studies}

This is the first study to explore perceived influences on HCPs' behaviours related to follow-up care post-TIA/ minor stroke, specifically identification and management of patients' needs. In stroke research, HCPs' perceived roles and beliefs about consequences have been found to influence rehabilitation assessment and referral practices on Australian acute stroke units [19]. Similarly, in primary care, research shows disparity in GPs' perception of their role in cardiovascular disease prevention, in particular lifestyle advice, and patient factors were important influences on GPs actions [20, 21]. We identified sub-optimal and variable interface communication 
between healthcare settings, which is supported by other stroke research [22]..

Lack of adequate follow-up is reflected in studies exploring perspectives of follow up from people with TIA and minor stroke, which found these individuals feel abandoned post-discharge [5]; frustrated due to lack of recognition of problems from HCP [23]; and dissatisfied with the lack of communication [24], holistic care [25], rehabilitation options [10], and individualised information and support [24]. Knowledge of stroke and medication has been identified as a barrier to secondary stroke prevention medication adherence; therefore, inadequate follow-up care adversely affects both patients satisfaction with care and clinical outcomes [26]..

\section{Implications for clinicians and policy makers}

Research has shown that structured stroke care at the post-acute stage is essential to improve secondary prevention post-stroke [27-30]. However, holistic care should also incorporate management of residual problems and education about diagnosis and stroke risk. There is no clear follow-up pathway to support people after TIA/ minor stroke and HCPs have disparate views on their responsibility and role in follow-up, which may mean patients slip through the net and receive no follow-up. We have identified factors that influence HCPs actions related to follow-up care post-TIA/ minor stroke which can help inform guidelines to improve healthcare for these patients. A key recommendation is to develop a structured follow-up pathway which incorporates specialist stroke care from consultants, holistic care from stroke nurses/ AHPs and long-term care from primary care GPs/ nurses. HCPs roles within this pathway should be clearly defined (Social professional role and identity) to influence their Intentions and Goals.

\section{Future research}

Identification and management of needs post-TIA/minor stroke is currently sub-optimal. Through identifying TDF domains relevant these behaviours, our findings provide an understanding of what needs to change to improve follow-up post-TIA/minor stroke and potential intervention targets. Future research should use this knowledge to inform intervention development. The Behaviour Change Wheel [31], a framework for behaviour change intervention design, can be used to systematically link the relevant TDF domains to 'intervention functions. For example, the TDF domain 'knowledge' (lack of knowledge of TIA/minor stroke patients' needs) maps to the intervention function 'education. Multiple intervention functions can be included in a complex intervention to change HCPs' behaviour. Previous follow-up interventions post-TIA/minor stroke have focused on secondary prevention medication and lifestyle change [28]; however, it is important for future interventions to address holistic needs [5].

\section{Strengths and limitations}

A strength of our study is that we drew upon a wide range of HCPs experiences across different healthcare settings and disciplines. These different perspectives enriched our understanding of the diverse range factors influencing follow-up care across different settings. Through use of TDF, we used behavioural theory to identify influences on behaviour (i.e. identifying and addressing needs post-TIA/ minor stroke), which are potentially amenable to change and could be targeted to improve care. An advantage of TDF is that it links to the Behaviour Change Wheel which provides a methodology for progressing to developing behaviour change intervention [17]. However, a limitation of collecting TDF data though interview is that we can only describe participants' reported influences on care after TIA and minor stroke and such perceptions do not necessarily fully reflect actual influences. A limitation of our study is that, for pragmatic reasons, only a subset of transcripts were double coded. Although it was not the purpose of our study to be generalisable, we only sampled participants from the West Midlands. Therefore, future research could explore if our findings are consistent across other geographical locations.

\section{Conclusion}

Following rapid discharge from hospital, people with TIA or minor stroke have limited access to follow-up care and care they do receive is inadequate. Through identifying factors which influence follow-up care of these patients, we can inform guidelines and practical strategies to improve holistic healthcare post-TIA and minor stroke.

\section{Abbreviations \\ AHP: Allied health professionals; GPs: General practitioners; HCP: Healthcare providers; TDF: Theoretical Domains Framework; TIA: Transient ischaemic attack.}

\section{Supplementary Information}

The online version contains supplementary material available at https://doi. org/10.1186/s12913-022-07607-0.

Additional file 1: eTable 1. Theoretical Domains Framework (TDF) version 2. eTable 2. Characteristics of healthcare provider participants.

\section{Acknowledgements}

Thank you to our patient partners who provided extremely valuable feedback and insight into the study design and interpretation of results. We thank also all participants who contributed to this study. 


\section{Authors' contributions}

GMT, MC, JM, RF and LA contributed to the study conception and design. GMT conducted the interviews and data were analysed by GMT and RA. GMT, RA, MC, JM, RF and LA were involved in the interpretation of results. GMT drafted the manuscript and RA, MC, JM, RF and LA provided feedback. All authors read and approved the final manuscript.

\section{Funding}

GT is funded by a National Institute for Health Research (NIHR) Postdoctoral Fellowship Award. This article/paper/report presents independent research funded by the NIHR (and Health Education England if applicable). MC receives funding from the NIHR Birmingham Biomedical Research Centre, NIHR Surgical Reconstruction and Microbiology Research Centre at the University Hospitals Birmingham NHS Foundation Trust and the University of Birmingham, Health Data Research UK, Innovate UK and Macmillan Cancer Support. JM is an NIHR Senior Investigator. The views expressed are those of the author(s) and not necessarily those of the NHS, the NIHR or the Department of Health. The funding bodies played no role in the design of the study and collection, analysis, and interpretation of data and in writing the manuscript.

\section{Availability of data and materials}

The datasets used and/or analysed during the current study available from the corresponding author on reasonable request.

\section{Declarations}

\section{Ethics approval and informed consent to participate}

Ethical approval was given by the Warwickshire North West - Greater Manchester East Research Ethics Committee (Reference: 17/NW/0737). Written or recorded verbal informed consent was obtained from the participants, by the interviewer, immediately prior to the interview.

\section{Consent for publication}

Not applicable.

\section{Competing interests}

MC is a National Institute for Health Research (NIHR) Senior Investigator and receives funding from the National Institute for Health Research (NIHR) Birmingham Biomedical Research Centre, the NIHR Surgical Reconstruction and Microbiology Research Centre and NIHR ARC West Midlands at the University of Birmingham and University Hospitals Birmingham NHS Foundation Trust, UKRI, Health Data Research UK, Innovate UK (part of UK Research and Innovation), Macmillan Cancer Support, UCB Pharma and GSK. MC has received personal fees from Astellas, Takeda, Merck, Daiichi Sankyo, Glaukos, GSK and the Patient-Centered Outcomes Research Institute (PCORI) outside the submitted work. The views expressed in this publication are those of the author(s) and not necessarily those of the NIHR or the Department of Health and Social Care. GMT, RA, RF, JM and LA declare no conflict of interest.

\section{Author details \\ ${ }^{1}$ Institute of Applied Health Research, University of Birmingham, B15 2TT, Birmingham, UK. ${ }^{2}$ Centre for Patient Reported Outcomes Research, University of Birmingham, B15 2TT, Birmingham, UK. ${ }^{3} \mathrm{NIHR}$ Surgical Reconstruction and Microbiology Research Centre, University Hospitals Birmingham NHS Foundation Trust and University of Birmingham, B15 2TH, Birmingham, UK. ${ }^{4}$ Population Health Sciences Institute, Faculty of Medical Sciences, Newcastle University, Newcastle upon Tyne NE1 7RU, UK. ${ }^{5}$ Primary Care Unit, Department of Public Health and Primary Care, University of Cambridge, Cambridge CB1 $8 \mathrm{RN}$, UK. ${ }^{6}$ Centre for Behaviour Change, University College London, WC1E 6BT, London, UK. ${ }^{7}$ Leeds Institute for Health Sciences, University of Leeds, Leeds LS2 9JT, UK. ${ }^{8}$ NIHR Birmingham Biomedical Research Centre, University Hospitals Birmingham NHS Foundation Trust and University of Birmingham, B15 2TH, Birmingham, UK. ${ }^{9}$ Birmingham Health Partners Centre for Regula- tory Science and Innovation, University of Birmingham, B15 2TT, Birmingham, UK. ${ }^{10} \mathrm{NIHR}$ Applied Research Collaboration (ARC) West Midlands, University of Birmingham, B15 2TT, Birmingham, UK.}

Received: 11 October 2021 Accepted: 27 January 2022

Published online: 21 February 2022

\section{References}

1. Scarborough P, Peto V, Bhatnagar P, Kaur A, Leal J, Luengo-Fernandez $\mathrm{R}$, et al. Stroke statistics. London: British Heart Foundation and Stroke Association; 2009.

2. European Stroke Organisation (ESO) Executive Committee. Guidelines for management of ischaemic stroke and transient ischaemic attack 2008. Cerebrovasc Dis (Basel, Switzerland). 2008;25(5):457-507.

3. Intercollegiate Stroke Working Party. National clinical guideline for stroke. London: Royal College of Physicians; 2016.

4. Kernan WN, Ovbiagele B, Black HR, Bravata DM, Chimowitz MI, Ezekowitz $M D$, et al. Guidelines for the prevention of stroke in patients with stroke and transient ischemic attack. Stroke. 2014;45(7):2160-236.

5. Turner GM, McMullan C, Atkins L, Foy R, Mant J, Calvert M. TIA and minor stroke: a qualitative study of long-term impact and experiences of followup care. BMC Fam Pract. 2019;20(1):176.

6. Moran GM, Fletcher B, Feltham MG, Calvert M, Sackley C, Marshall T. Fatigue, psychological and cognitive impairment following transient ischaemic attack and minor stroke: a systematic review. Eur J Neurol. 2014;21(10):1258-67.

7. Sackley CM, Mant J, McManus RJ, Humphreys G, Sharp L, Mares K, et al. Functional and emotional outcomes after transient ischemic attack: a 12-month prospective controlled cohort study. Int J Stroke. 2019;14(5):522-529.

8. Turner GM, Calvert M, Feltham MG, Ryan R, Marshall T. Ongoing impairments following transient ischaemic attack: retrospective cohort study. Eur J Neurol. 2016;23(11):1642-50.

9. van Rooij FG, Kessels RP, Richard E, De Leeuw FE, van Dijk EJ. Cognitive Impairment in Transient Ischemic Attack Patients: A Systematic Review. Cerebrovasc Dis (Basel, Switzerland). 2016;42(1-2):1-9.

10. Carlsson GE, Moller A, Blomstrand C. Managing an everyday life of uncertainty--a qualitative study of coping in persons with mild stroke. Disabil Rehabil. 2009;31(10):773-82.

11. Fride Y, Adamit T, Maeir A, Ben Assayag E, Bornstein NM, Korczyn AD, et al. What are the correlates of cognition and participation to return to work after first ever mild stroke? Top Stroke Rehabil. 2015;22(5):317-25.

12. Hommel M, Trabucco-Miguel S, Joray S, Naegele B, Gonnet N, Jaillard A. Social dysfunctioning after mild to moderate first-ever stroke at vocational age. J Neurol Neurosurg Psychiatry. 2009;80(4):371-5.

13. Muus I, Petzold M, Ringsberg KC. Health-related quality of life among Danish patients 3 and 12 months after TIA or mild stroke. Scand J Caring Sci. 2010;24(2):211-8.

14. Verbraak ME, Hoeksma AF, Lindeboom R, Kwa VI. Subtle problems in activities of daily living after a transient ischemic attack or an apparently fully recovered non-disabling stroke. J Stroke Cerebrovasc Dis. 2012;21(2):124-30.

15. Wang YL, Pan YS, Zhao XQ, Wang D, Johnston SC, Liu LP, et al. Recurrent stroke was associated with poor quality of life in patients with transient ischemic attack or minor stroke: finding from the CHANCE trial. CNS Neurosci Therapeut. 2014;20(12):1029-35.

16. Ravenhill G, Gkanasouli L, Lawrence M. Experiences of transient ischaemic attack diagnosis and secondary prevention: a qualitative review. British J Neurosci Nurs. 2019;15(Sup5):S14-25.

17. Atkins $L$, Francis J, Islam R, O'Connor D, Patey A, Ivers N, et al. A guide to using the theoretical domains framework of behaviour change to investigate implementation problems. Implement Sci. 2017;12(1):77.

18. Woods M, Macklin R, Lewis GK. Researcher reflexivity: exploring the impacts of CAQDAS use. Int J Soc Res Methodol. 2016;19(4):385-403.

19. Lynch EA, Luker JA, Cadilhac DA, Fryer CE, Hillier SL. A qualitative study using the theoretical domains framework to investigate why patients were or were not assessed for rehabilitation after stroke. Clin Rehabil. 2017;31(7):966-77.

20. Ju I, Banks E, Calabria B, Ju A, Agostino J, Korda RJ, et al. General practitioners' perspectives on the prevention of cardiovascular disease: systematic review and thematic synthesis of qualitative studies. BMJ Open. 2018;8(11):e021137.

21. Lawton R, Heyhoe J, Louch G, Ingleson E, Glidewell L, Willis TA, et al. Using the theoretical domains framework (TDF) to understand adherence to multiple evidence-based indicators in primary care: a qualitative study. Implement Sci. 2016;11(1):113.

22. Pedersen RA, Petursson $H$, Hetlevik I, Thune $H$. Stroke follow-up in primary care: a discourse study on the discharge summary as a tool for knowledge transfer and collaboration. BMC Health Serv Res. 2021;21(1):41. 
23. Taule T, Raheim M. Life changed existentially: a qualitative study of experiences at 6-8 months after mild stroke. Disabil Rehabil. 2014;36(25):2107-19.

24. Hillsdon KM, Kersten P, Kirk HJ. A qualitative study exploring patients' experiences of standard care or cardiac rehabilitation post minor stroke and transient ischaemic attack. Clin Rehabil. 2013;27(9):845-53.

25. Croot EJ, Ryan TW, Read J, Campbell F, O'Cathain A, Venables G. Transient ischaemic attack: a qualitative study of the long term consequences for patients. BMC Fam Pract. 2014:15:174.

26. Jamison J, Graffy J, Mullis R, Mant J, Sutton S. Barriers to medication adherence for the secondary prevention of stroke: a qualitative interview study in primary care. Br J Gen Pract. 2016;66(649):e568-e76.

27. Bridgwood B, Lager KE, Mistri AK, Khunti K, Wilson AD, Modi P. Interventions for improving modifiable risk factor control in the secondary prevention of stroke. Cochrane Database Syst Rev. 2018;5(5):Cd009103.

28. Ahmadi M, Laumeier I, Ihl T, Steinicke M, Ferse C, Endres M, et al. A support programme for secondary prevention in patients with transient ischaemic attack and minor stroke (INSPiRE-TMS): an open-label, randomised controlled trial. Lancet Neurol. 2020;19(1):49-60.

29. Willeit P, Toell T, Boehme C, Krebs S, Mayer L, Lang C, et al. STROKE-CARD care to prevent cardiovascular events and improve quality of life after acute ischaemic stroke or TIA: a randomised clinical trial. EClinicalMedicine. 2020;25:100476.

30. Ullberg T, Månsson K, Berhin I, Pessah-Rasmussen H. Comprehensive and structured 3-month stroke follow-up using the post-stroke checklist (the Struct-FU study): a feasibility and explorative study. J Stroke Cerebrovasc Dis. 2021:30(2):105482.

31. Michie S, van Stralen MM, West R. The behaviour change wheel: a new method for characterising and designing behaviour change interventions. Implement Sci. 2011;6(1):42.

\section{Publisher's Note}

Springer Nature remains neutral with regard to jurisdictional claims in published maps and institutional affiliations.

- fast, convenient online submission

- thorough peer review by experienced researchers in your field

- rapid publication on acceptance

- support for research data, including large and complex data types

- gold Open Access which fosters wider collaboration and increased citations

- maximum visibility for your research: over 100M website views per year

At BMC, research is always in progress.

Learn more biomedcentral.com/submissions 